TOMASZ SWOBODA

ORCID: 0000-0002-8285-3837

Université de Gdańsk

tomasz.swoboda@ug.edu.pl

\title{
VERS LES FONDEMENTS ANTHROPOLOGIQUES DU POST-SÉCULARISME : L'EXEMPLE DU COLLÈGE DE SOCIOLOGIE
}

Le nom de Georges Bataille s'impose à quiconque veut réfléchir sur la post-sécularité avant la lettre, et pas seulement en France. Son grand projet de La Somme athéologique, inachevé, imprécis ou, pour utiliser une notion chère à Bataille, informe, mais dont le titre même en dit long sur les visées de son auteur, occupe une place à part dans l'histoire de la pensée occidentale. Résultat d'un long parcours, à la fois méthodique et personnel, à travers l'histoire de la philosophie et l'histoire tout court, les textes qui composent La Somme, mais aussi d'autres essais et récits de Bataille, toujours marqués par un rapport particulier au religieux et au sacré, proposent des points de vue, sinon convaincants, du moins intéressants, sur des problèmes débattus ces dernières années autour de la question de la post-sécularité, tels que le rapport de l'immanence et de la transcendance, celui de l'humain et du divin, ou encore la place du sacré dans la vie individuelle et sociale.

Ce dernier problème, abordé abondamment dans les écrits de Bataille des années 1940, avait fait l'objet de sa réflexion dès la décennie précédente, et ce non seulement à titre personnel mais aussi dans le cadre de l'entreprise collective que fut le Collège de Sociologie. Fondé par Bataille avec Roger Caillois et Michel Leiris, et conçu comme une réaction des intellectuels parisiens de gauche contre le fascisme, le Collège se prête parfaitement à une analyse à la lumière de la pensée post-séculière dans la mesure où il représente différents aspects de celle-ci : a-théologique chez Bataille, communautaire chez Caillois, intime chez 
Leiris. Après avoir examiné chacun de ces aspects, nous étudierons les formes que les concepts du religieux et du politique, ici inextricablement liés, revêtent dans le discours du Collège, pour réfléchir enfin sur les fondements anthropologiques d'un retour original au sacré que proposait cette institution éphémère.

En effet, c'est la concentration sur le sacré au sens large qui permet de considérer les conceptions créées au sein du Collège comme apparentées à l'attitude post-séculière, si l'on admet que le contexte socio-politique de la France des années 1930 correspond à ce que Charles Taylor définit comme " l'ère laïque », c'est-àdire une époque où la religion cesse d'être un élément obligatoire et sous-entendu de la vision du monde pour devenir seulement une des options possibles ${ }^{1}$, et que la sécularisation qui en découle couvre les trois champs : politique, social et individuel $^{2}$. Le point de vue des membres du Collège sur le sacré s'inscrit aussi dans ce qui apparaît comme une caractéristique principale du post-sécularisme, mise en relief dès la première description du phénomène par John McClure, à savoir le refus de choisir entre la religion et la laïcité ${ }^{3}$. Considéré comme théoricien majeur du post-sécularisme, Jürgen Habermas parle de son côté de « la continuation vigoureuse de la religion dans un environnement de plus en plus sécularisé »4, alors que pour Agata Bielik-Robson, la plus grande figure du post-sécularisme en Pologne, le trait constitutif de celui-ci consiste à « se maintenir entre les deux » : il ne s'agirait ni d'un retour de la foi, ni de l'effacement de la trace de la transcendance ${ }^{5}$, mais d'une via media à laquelle consentiraient sans doute, bien qu'à différents degrés, Bataille, Caillois et Leiris.

La place d'Habermas, représentant contemporain de l'École de Francfort, dans l'histoire de la pensée post-séculière incite même à chercher une relation de filiation entre lui et le Collège, étant donné que parmi les sympathisants de celui-ci se trouvait l'un des fondateurs de l'École de Francfort, Walter Benjamin. Cependant, au lieu d'aller dans ce sens-là, nous essaierons de suivre un chemin proche de celui indiqué par Michał Warchala dans son étude sur la religion romantique, où le chercheur polonais retrace une " généalogie de la pensée post-séculière $»^{6}$.

${ }^{1}$ Voir C. Taylor, Secular Age, Harvard University Press, Cambridge 2007.

2 C.M. Gómez, «Las condiciones postseculares de la creencia religiosa », [dans :] La religión en la sociedad postsecular: transformación y relocalización de lo religioso en la modernidad tardía, C.M. Gómez (dir.), Universidad del Rosario, Bogotá 2014, pp. 27-31.

3 J.A. McClure, « Post Secular Culture: The Return of Religion in Contemporary Theory and Literature », Cross Currents 47/3, 1997, p. 340.

4 J. Habermas, « Equal Treatment of Cultures and the Limits of Postmodern Liberalism », Journal of Political Philosophy 13/1, 2005, p. 26.

${ }^{5}$ A. Bielik-Robson, «Deus otiosus: ślad, widmo, karzeł», [dans :] Deus otiosus. Nowoczesność w perspektywie postsekularnej, A. Bielik-Robson, M.A. Sosnowski (dir.), Wydawnictwo Krytyki Politycznej, Warszawa 2013, pp. 10-11.

${ }^{6} \mathrm{M}$. Warchala, « Religia romantyczna. Z genealogii myślenia postsekularnego », [dans :] Deus otiosus. Nowoczesność w perspektywie postsekularnej, pp. 167-187. 
Le Collège de Sociologie n'a existé que deux ans, de novembre 1937 à juillet 1939, sous la forme d'une institution mi-officielle, mi-privée, dont le fonctionnement consistait à tenir des conférences toutes les deux ou trois semaines au fond d'une librairie parisienne. Conçu dans le milieu des intellectuels parisiens de gauche - une gauche plutôt communiste que socialiste, plutôt anarchiste que réformiste - , au centre duquel se trouvait Georges Bataille, employé de la Bibliothèque Nationale, ancien rédacteur de la revue iconoclaste Documents, le Collège était une conséquence logique des engagements politiques de celui-ci, y compris dans la Contre-Attaque, mouvement révolutionnaire dans le cadre duquel Bataille s'était réconcilié avec son ennemi de longue date André Breton. Effrayés par la montée du fascisme et déçus par l'impuissance des démocraties européennes, en premier lieu du gouvernement du Front Populaire, les concepteurs du Collège ont décidé de chercher les solutions ailleurs.

Le nom même du " collège », qui connote — comme le souligne Denis Hollier, auquel on doit la publication de la plupart des documents relatifs à cette institution - « une organisation collégiale, une cléricature $»^{7}$, montre la direction dans laquelle voulaient s'engager les signataires de la « Déclaration sur la fondation d'un Collège de Sociologie »: Georges Ambrosino, Georges Bataille, Georges Caillois, Pierre Klossowski, Pierre Libra et Jules Monnerot. En effet, guidés par les idées de la sociologie durkheimienne ainsi que par l'ethnologie et l'ethnographie nouvelles — dont ils étaient en partie des adeptes - les fondateurs du Collège ont non seulement dirigé les travaux de celui-ci vers le modèle de la « société des hommes » mais le Collège lui-même a emprunté à ce modèle, du moins partiellement, sa forme et son organisation. "Nous rêvions, dira plus tard Caillois, d'associations occultes et toutes-puissantes, d'ordres à la fois militaires et religieux, tout ceci avec un arrière-plan parfois très proche d'initiations rituelles et de sacrifices humains consentis pour donner à la conspiration un départ décisif $»^{8}$. Rien d'étonnant donc à ce que le Collège ait été, au dire de Klossowski, une " émanation exotérisante du groupe fermé et secret d'Acéphale ${ }^{9}$, formé par Bataille autour du rite du sacrifice humain et de l'idée religieuse de la communauté. Dans la « Conclusion annuelle » de l'activité d'Acéphale, en 1937, Bataille définit d'ailleurs le Collège naissant comme un essai de « donner à cette entreprise [Acéphale] une base théorique sur le plan de la connaissance la mieux contrôlée ${ }^{10}$.

${ }^{7}$ D. Hollier, « À l'en-tête d'Acéphale », [dans :] Le Collège de Sociologie 1937-1939, D. Hollier (dir.), Gallimard, Paris 1995, p. 11.

${ }^{8}$ R. Caillois, « Notes pour un itinéraire de Roger Caillois », [dans :] Cahiers pour un temps : Roger Caillois, Centre Georges Pompidou / Pandora Éditions, Paris 1981, pp. 166-167.

${ }^{9}$ Cité d'après Le Collège de Sociologie..., p. 884.

${ }^{10}$ G. Bataille, «Conclusion annuelle, 24 septembre 1937 », [dans :] L'Apprenti Sorcier. Textes, lettres et documents (1932-1939), M. Galletti (dir.), La Différence, Paris 1999, p. 405. 
Fondateur et membre le plus actif du Collège, Bataille intervient dans le cadre de celui-ci à plusieurs reprises. Il donne notamment des conférences sur « la sociologie sacrée », « l'attraction et la répulsion », « la structure et la fonction de l'armée », " Hitler et l'Ordre teutonique » et « la joie devant la mort », avant de mettre fin aux activités de l'institution et de poursuivre ses réflexions sur un plan personnel, et non plus collectif. En effet, il semble que l'expérience du Collège de Sociologie - ainsi que celle d'Acéphale - ait permis à Bataille de développer ses conceptions du sacré et de la religion, de les rendre plus cohérentes pour enfin les exprimer dans des ouvrages tels que L'Expérience intérieure, Le Coupable et Sur Nietzsche. Ainsi Bataille a-t-il réussi à harmoniser des apports extrêmement hétérogènes provenant de ses lectures : comme le rappelle Francis Marmande, dans les années 1930,

Bataille lit nombre de livres portant sur l'Inde, les religions, la Chine, les voyages en Orient, les techniques de yoga, le bouddhisme, etc. Cette bibliothèque se mélange aux emprunts de la psychanalyse, philosophie politique, histoire des religions et d'ouvrages relatifs aux perversions, à la sexualité, à l'histoire de la folie ${ }^{11}$.

Cet amalgame de sources débouche sur une idée originale du sacré qui se passe de Dieu et met l'accent sur l'expérience de l'excès, de la mort, de la destruction, mais aussi de la communauté et de la joie. « La joie catastrophique de Bataille en tant que force affective qui résiste à l'attraction normative de tout transcendant au-delà, qu'il soit religieux ou laïque $»^{12}$, ne prend Dieu en considération que dans la mesure où il est nécessaire à ce que Agata Bielik-Robson considère comme élément constitutif de l'attitude post-séculière, à savoir la « spéculation métaphysique », qui peut concerner un Dieu absent, un Dieu mort, un Deus absconditus ou un Deus otiosus ${ }^{13}$.

La transgression, notion que Bataille ne théorisera pleinement que quelques années plus tard, est déjà implicitement présente dans ses interventions « collégiales ", étant donné que la recherche des forces aptes à s'opposer au pouvoir mythique du fascisme est, dans la plupart des cas, menée du côté des interdits, des excès et de ces éléments du réel qu'il se proposait d'étudier dans le cadre de «l'hétérologie », c'est-à-dire d'une « science de ce qui est tout autre » ${ }^{14}$ : ordures, matières excrémentielles, parties sexuelles du corps, etc. Il s'agit d'une recherche qui s'effectue dans un mouvement communiel où c'est la relation avec l'autre, et non pas cet autre lui-même, qui constitue l'objectif des démarches, à la fois sur le plan théorique et existentiel. Comme le dit Paul Hegarty :

${ }^{11}$ F. Marmande, Le pur bonheur : Georges Bataille, Nouvelles Éditions Lignes, Paris 2011, p. 285.

12 A. Dubilet, « The Catastrophic Joy of Abandoning Salvation: Thinking the Postsecular with Georges Bataille », Journal for Cultural and Religious Theory 16/2, 2017, p. 164.

13 A. Bielik-Robson, op. cit., pp. 7-8.

${ }^{14}$ G. Bataille, CEuvres complètes, t. 2, Gallimard, Paris 1972, p. 59. 
le sacré de Bataille diffère du sacré tel qu'on le conçoit traditionnellement en ce qu'il n'a pas d'espace ; il apporte plutôt un espace pour lui-même, par la transgression (rappelant que le sacré est lui-même une transgression du profane et vice versa). En conséquence, le sacré n'est jamais, il n'est jamais pleinement - ce que nous avons à la place est une tentative de donner le sacré comme un don ${ }^{15}$.

L'absence étant, chez Bataille, non seulement un fond sur lequel peut avoir lieu « l'expérience intérieure » mais aussi la condition sine qua non de celle-ci, la recherche ou la recréation du sacré s'approche inévitablement de la mystique : une mystique sans Dieu, bien sûr, qui débouche sur une « nouvelle théologie (qui n'a que l'inconnu pour objet) $»^{16}$.

Quant à Roger Caillois, qui co-dirigeait avec Bataille les activités du Collège et assurait une bonne partie des interventions, ses idées relatives à la religion allaient dans un sens complètement différent. Né en 1913 et donc beaucoup plus jeune que la plupart des membres du Collège, mais déjà auteur brillant du Mythe et l'homme et collaborateur des plus prestigieuses revues parisiennes, Caillois se distingue dès le début de sa carrière - aussi au sein du Collège — par son rationalisme poussé à l'extrême. Dans ses conférences sur " les sociétés animales », les " confréries, ordres, sociétés secrètes, églises », « l'ambiguïté du sacré » ou « la sociologie du bourreau », il reste moins, contrairement à Bataille, sous l'emprise des phénomènes étudiés qu'il n'essaie de percer leurs mécanismes de fonctionnement — de même qu'il l'a fait, jeune adepte du surréalisme, dans l'épisode fameux des « haricots sauteurs » où, au lieu d'admirer les grains bondissants, il a proposé de les couper pour vérifier s'ils ne renfermaient pas un insecte, ce qui a provoqué sa rupture avec le mouvement ${ }^{17}$. Même dans son intervention la plus controversée, intitulée "Le vent d'hiver », où il s'abandonne parfois à une espèce d'expressionnisme politique militant, ce qui lui vaut - à lui et au Collège tout entier - des accusations de " sur-fascisme », Caillois reste « anticlérical, athée, - quoique captivé par le sacré —, antimarxiste $»^{18}$ mais avant tout un intellectuel rigoureux qui cherche un moyen pour détruire l'ordre ancien et en instaurer un nouveau. C'est pourquoi, comme le dit Denis Hollier, aux yeux de Caillois, " le Collège doit devenir une sorte d'Ordre sociologique, au sens où on parle d'Ordre teutonique, un noyau dense à partir duquel se propagerait un complot, une conjuration $»^{19}$.

Bataille mystique, Caillois politique : Leiris est autobiographique. Beaucoup moins engagé dans les travaux du Collège que ses deux amis, l'auteur de L'Afrique fantôme fait néanmoins partie du grand trio de l'institution, d'abord par le lien personnel et intellectuel qui l'unit avec eux, ensuite par le fait que sa seule

15 P. Hegarty, «Undelivered: the Space/Time of the Sacred in Bataille and Benjamin », Economy and Society 32/1, 2003, p. 102.

16 G. Bataille, Euvres complètes, t. 5, Gallimard, Paris 1973, p. 120.

17 O. Felgine, Roger Caillois, Stock, Paris 1994, p. 101.

18 Ibidem, p. 158.

${ }^{19}$ Le Collège de Sociologie..., p. 218. 
intervention au Collège est publiée dans La Nouvelle revue française, à côté des textes de Bataille et Caillois, en tant qu'échantillon représentatif de l'entreprise. «Le sacré dans la vie quotidienne », lu dans la Salle des Galeries du Livre, 15, rue Gay-Lussac, le 8 janvier 1938, contient également un troisième aspect de la religion et du sacré abordé par les membres du Collège. Or, il aurait été naturel que Leiris, qui avait déjà derrière lui l'expérience de la revue Documents et, avant tout, la participation à la mission ethnographique Dakar-Djibouti, parle du sacré des sociétés dites primitives, dont il était l'un des meilleurs spécialistes. Cependant, il préfère rester sur la voie tracée par L'âge d'homme, déjà achevé mais publié seulement en 1939, c'est-à-dire sur la voie des mythes personnels éclairés par des moyens proches de la psychanalyse. Certes, dans son texte et dans ses notes préparatoires, il apparaît assez proche de Bataille quant aux modalités du sacré : « le sacré existera, écrit-il, non dans des choses ou des personnes, mais dans le rapport que j'entretiens avec certaines choses ou certaines personnes. Ainsi le sacré reste fluide, n'est jamais substantialisé $»^{20}$. Mais dans le même temps, Leiris rejette la vision rituelle et orgiaque du sacré de Bataille et, pour ainsi dire, enfreint la transgression de ce dernier en réduisant l'expérience du sacré à son aspect le plus personnel, qui se manifeste à travers les souvenirs d'enfance. Il rejette également l'enseignement d'Émile Durkheim et de Marcel Mauss, et « se situe, quant à la distinction sacré/profane, plus près d'une quête proustienne que d'un effort pour confirmer la théorie sociologique de ses maîtres $»^{21}$. Le sacré ordinaire et quotidien, un sacré non seulement sans Dieu mais aussi sans divinité aucune : telle est la proposition à la fois théorique et pratique de Leiris quant aux moyens de remédier à l'expansion des pouvoirs telluriques qui sévissent en Europe. Profondément sceptique et mélancolique, cette proposition montre toutefois un des possibles de la mentalité post-séculière avant la lettre.

Ici du moins, dans cette attitude imprégnée de résignation, Leiris est rejoint par Bataille qui, dans son rapport au sacré, fait montre d'une «sensibilité tragique », contrairement à Caillois, pour qui les figures traditionnelles du sacré devaient servir à acquérir une «maitrise de soi $»^{22}$. En général, les conceptions du trio quant aux aspects religieux de la vie, tantôt sociale, tantôt individuelle, divergent au point qu'il n'est pas possible d'y trouver de dénominateur commun, si ce n'est la fascination pour ces différents aspects du sacré. En commentant l'éclatement du Collège, la biographe de Leiris dit que cette institution

${ }^{20}$ M. Leiris, L'homme sans honneur. Notes pour un sacré dans la vie quotidienne, Jean-Michel Place, Paris 1994, p. 126.

${ }^{21}$ J.-P. Colleyn, «L'informe, le sacrilège et le passage à l'art. Leiris, le boli et le sacré », [dans :] Leiris unlimited, D. Hollier, J. Jamin (dir.), CNRS éditions, Paris 2017, p. 105.

${ }^{22}$ Termes utilisés par Simonetta Falasca-Zamponi dans son livre Rethinking the Political: The Sacred, Aesthetic Politics, and the Collège de Sociologie, McGill-Queen's University Press, Montreal-Kingston 2011, pp. 209 et 215. 
aura été une tentative de concilier des points de vue par trop contradictoires : celui de Michel Leiris qui attache une importance particulière au sacré dans la vie quotidienne, celui de Bataille dont l'ambition, à travers le Collège, est de comprendre les structures sociales pour mieux les transformer et fonder une communauté morale nouvelle, celui de Caillois qui cherche à élaborer les moyens d'une volonté politique ${ }^{23}$.

Il n'en reste pas moins que le Collège lui-même, en tant qu'entreprise intellectuelle, constitue un exemple intéressant d'institution en quelque sorte post-séculière, et les discordances entre Bataille, Caillois et Leiris ne font qu'enrichir son image on ne peut plus complexe ainsi que les conceptions du religieux et du sacré qui en découlent.

Anne Pibarot observe, à juste titre, que le Collège de Sociologie a été « à la limite de constituer une société secrète, un groupe d'initiés, bref de se doter, au-delà de la sphère intellectuelle, d'une dimension religieuse et activiste $»^{24}$. Denis Hollier, de son côté, évoque un masque «mi-guerrier, mi-religieux » de l'institution ${ }^{25}$. C'est que, pour ce qui est de la religion, le Collège s'en occupe plutôt comme d'une forme sous laquelle peuvent se manifester les liens sociaux - y compris ceux entre les membres du Collège lui-même — que comme d'un contenu philosophique ou théologique ${ }^{26}$. Si religion il y a, elle correspond à la tradition de la « religiosité non-normative » qui s'étend depuis Joachim de Fiore jusqu'à Jacques Derrida en passant par Descartes, Kant, Hegel et Marx ${ }^{27}$. En effet, les membres du Collège évoquent la religion à plusieurs reprises mais ils ne le font que pour l'abandonner aussitôt au profit du sacré, catégorie qui les intéresse au plus haut point, jusqu'à devenir l'axe principal de leurs travaux. Ce passage du religieux au sacré est manifeste dès la première conférence où Bataille tient à distinguer « la sociologie sacrée » de « la sociologie religieuse », celle-là pouvant « être considérée comme l'étude non seulement des institutions religieuses mais de l'ensemble du mouvement communiel de la société ${ }^{28}$. Mais cette distinction, essentielle pour comprendre la position du Collège, est peut-être le mieux exprimée par Leiris qui parle de la " nécessité d'étudier le sacré "à l'état naissant" plutôt que dans ses formes pétrifiées (religion, patrie, morale, etc.) qui sont du sacré droit dans la mesure même où elles sont codifiées et représentent donc l'ordre,

23 A. Armel, Michel Leiris, Fayard, Paris 1997, pp. 388-389.

24 A. Pibarot, Michel Leiris. Des premiers écrits à « L'Âge d'homme », Théétète, Nîmes 2004, p. 142.

25 D. Hollier, op. cit., p. 12.

${ }^{26}$ Comme nous l'avons observé ailleurs, « la religion qui sous-tend le discours du Collège y apparaît plutôt au niveau du signifiant » (T. Swoboda, Histoires de l'aeil, Rodopi, Amsterdam-New York, 2013, p. 171).

27 Voir A. Bielik-Robson, op. cit., pp. 11-12.

${ }^{28}$ G. Bataille, « La sociologie sacrée et les rapports entre "société", "organisme" et "être" », [dans :] Le Collège de Sociologie..., p. 36. 
la règle, la norme idéale », la religion elle-même correspondant à un « maximum de galvaudage » et à une « domestication du sacré $»^{29}$.

Il ne s'agit donc aucunement d'un retour à la religion mais d'une recréation du sacré dans une société profane, tout en espérant être personnellement atteint par les forces ainsi suscitées. Le troisième point de « La déclaration de la fondation d'un Collège de Sociologie » le dit clairement : «L'objet précis de l'activité envisagée peut recevoir le nom de sociologie sacrée, en tant qu'il implique l'étude de l'existence sociale dans toutes celles de ses manifestations où se fait jour la présence active du sacré ${ }^{30}$. C'est peut-être le caractère «total» de cette déclaration qui mérite une attention particulière, dans la mesure où non seulement dans ce texte, mais aussi dans les travaux effectifs du Collège, sont soumises à l'examen des formes extrêmement diverses du sacré, comme si le but de ces analyses consistait à mettre en relief cette diversité ou, plus précisément, l'ambiguïté même du sacré, ou bien encore, pour utiliser les termes de Bataille, « l'attraction et la répulsion » qu'il suscite. En effet, à l'encontre des religions officielles qui identifient le sacré avec «l'ordre, la règle, la norme idéale », les recherches menées au sein du Collège se tournent vers l'anthropologie - celle de Mauss notamment - pour rendre compte de « cette contradiction intolérable qui définit le sacré : l'extase divine et l'horreur extrême $»^{31}$. L'idée d'hétérologie déjà élaborée par Bataille, les lectures savantes de l'élève de Mauss qu'est Roger Caillois, l'expérience ethnographique de Leiris sont autant d'éléments qui permettent aux membres du Collège de réduire en cendres la conception d'un sacré uniforme, " galvaudé », pour revenir à l'ambivalence du phénomène. Plutôt qu'à un retour à la religion, on aurait donc affaire, au sein du Collège de Sociologie, à un retour à la polyvalence du sacré par le biais de l'anthropologie. C'est là peut-être que réside l'importance du Collège, et de Bataille en premier lieu, pour la pensée post-séculière sur le plan philosophique. Alex Dubilet résume très bien cette dimension de l'apport de Bataille en disant que

si sa pensée cible l'un des piliers centraux de l'ontologie séculière, le sujet auto-possédé, elle ne le fait pas à travers un appel à la transcendance mais en repensant radicalement le statut de l'immanence et de la transcendance, et en les séparant de leurs associations sémantiques traditionnelles, qui alignent l'immanence avec la laïcité et la transcendance avec la religion ${ }^{32}$.

Qui plus est, la fonction même de la transcendance et de la religion subit, au sein du Collège, une transformation significative. Or, dans plusieurs interventions - notamment celles de Caillois et Bataille - , ces deux instances se trouvent promues au rang d'une alternative à la politique. Cependant, ce détournement ne laisse pas la transcendance et la religion indemnes. En effet, le politique se

${ }^{29}$ M. Leiris, op. cit., pp. 93, 130, 125.

${ }^{30}$ Le Collège de Sociologie..., p. 27.

31 J. Biles, Ecce Monstrum. Georges Bataille and the Sacrifice of Form, Fordham University Press, New York 2007, p. 8.

32 A. Dubilet, op. cit., p. 164. 
glisse imperceptiblement dans le sacré, rapprochant dangereusement l'intérêt des membres du Collège pour le rituel social et les émotions collectives nées de l'esthétisation de la politique telle qu'elle était pratiquée par les fascistes. Denis Hollier rappelle à ce propos le séjour de Denis de Rougemont en Allemagne. L'auteur de L'Amour et l'Occident, qui a prononcé au Collège une conférence sur les " Arts d'aimer et arts militaires ", raconte dans son Journal d'Allemagne l'atmosphère qui accompagnait un discours d'Hitler ainsi que ses propres impressions : « $\mathrm{Ce}$ que j'éprouve à présent, dit-il, c'est cela qu'on doit appeler horreur sacrée $»^{33}$. Il n'y a là, semble-t-il, aucune métaphorisation ni hyperbolisation. Au contraire, la révélation de Rougemont, tout comme l'interchangeabilité du politique et du sacré chez Bataille et, surtout, chez Caillois, est une exemplification de ce que Harald Wydra, anthropologue de la politique, analyse comme l'identité du politique et du sacré, « forces jumelles », selon lui. Si, après la Révolution française, le sacré est passé de la sphère du pouvoir politique vers des pratiques culturelles, il n'en reste pas moins apparenté à ce pouvoir ou pour le moins à ces aspects esthétiques, tels que les commémorations et d'autres rituels collectifs ${ }^{34}$. Ainsi le Collège opère-t-il cette « collusion du politique et du religieux » dont parle Jean-Michel Besnier ${ }^{35}$, en mettant l'accent sur la dimension religieuse du pouvoir.

Certes, si l'on veut bien retrouver la signification de ce rétablissement d'un sacré « gauche », le contexte politique du moment n'est pas négligeable. Bernard Sichère rappelle, à juste titre, que « le travail du Collège a lieu dans une conjoncture réellement dramatique, celle du bellicisme hitlérien, des accords de Munich, de l'effondrement des démocraties, de la capitulation du gouvernement Daladier soutenu par les communistes $»^{36}$. Mais cette oscillation entre la politique et la religion, l'idée de la resacralisation du social à travers la violence mythique ou, pour parler en termes psychanalytiques, la sublimation politique de la sexualité en vue de transformer la société à travers une révolution totale, tous ces projets, explicites ou implicites, ne peuvent s'expliquer que comme une réaction à l'expansion du fascisme. Si aberrante et éloignée de la vérité politique qu'elle puisse paraître, l'idée de la ressemblance, sinon de la parenté, des conceptions du Collège et des forces que celui-ci voulait combattre semble partiellement justifiée, non pas sur le plan politique mais sur le plan anthropologique. En effet, les années 1930 sont profondément marquées par ce que Jean Clair appelle le passage du mécanomorphisme au biomorphisme ${ }^{37}$, non seulement dans le domaine de l'art

${ }^{33}$ Le Collège de Sociologie..., p. 406.

${ }^{34}$ Voir H. Wydra, Politics and the Sacred, Cambridge University Press, Cambridge 2015.

35 J.-M. Besnier, La politique de l'impossible. L'intellectuel entre révolte et engagement, La Découverte, Paris 1988, p. 110.

36 B. Sichère, Pour Bataille. Être, chance, souveraineté, Gallimard, Paris 2006, p. 91.

37 J. Clair, « La masse et la puissance. L'âge des dictatures ", [dans :] Les années 1930. La fabrique de "l'Homme nouveau », J. Clair (dir.), Musée des beaux-arts du Canada / Gallimard, Ottawa-Paris 2008, p. 18. 
mais aussi dans celui de la mentalité collective. L'eugénisme des États totalitaires, l'idée communiste de l'homme nouveau, la fascination pour les métamorphoses monstrueuses, la société considérée comme un organisme ou un corps, sont autant de formes sous lesquelles se manifeste le fantasme de la régénération, qui n'est pas non plus étranger aux membres du Collège de Sociologie. En effet, leur " sociologie sacrée » échappait, elle aussi, à l'image de la communauté fondée sur un « contrat social » pour céder la place à celle scellée par les rites et les sacrifices. Si Laura Bossi met en relief une « rivalité mimétique » des totalitarismes $\mathrm{du} \mathrm{XX}^{\mathrm{e}}$ siècle avec le christianisme, rivalité « qui poussa ces régimes totalitaires à développer des caractères de religion civile, de pseudo-religion ou de religion de substitution $»^{38}$, avec leur propre liturgie et leurs propres rites, il faut convenir que le Collège a voulu utiliser quasiment les mêmes armes. Peut-être sans vraiment le vouloir, si l'on en croit Jean Clair selon qui l'idée de créer « l'homme nouveau » non seulement « flottait dans l'air » des années 1930, mais encore dominait l'époque, indépendamment des régimes politiques et des traditions culturelles ${ }^{39}$.

La différence reste pourtant énorme. En effet, tout en étant lui-même formé à la fois comme un ordre religieux et un corps politique, comme une espèce de « religion civile », le Collège de Sociologie a soumis les phénomènes de ce type à une analyse politique, philosophique, psychanalytique et sociologique minutieuse, proposant ainsi une des premières théorisations des structures biopolitiques en Occident. Qui plus est, en mettant l'accent sur la dimension subversive, voire révolutionnaire, de la religion ou, plus largement, de la spiritualité, les membres du Collège permettent « une reconsidération minoritaire de la post-sécularité » ${ }^{40}$. Par ces deux aspects, ils précèdent et annoncent les recherches de Michel Foucault et Giorgio Agamben ${ }^{41}$ dont les écrits peuvent être relus à la lumière de cette conjonction de la post-sécularité et du nouveau langage du sacré tel qu'il a été conçu par le Collège.

C'est peut-être l'invention, ou du moins la recherche de ce nouveau langage et, à travers lui, du «nouvel homme » qui constitue l'apport le plus important du Collège de Sociologie à la discussion sur la post-sécularité. En passant du plan purement politique ou religieux à celui qui privilégie la réflexion sur l'interpénétration du corps et du pouvoir, Bataille et Leiris révèlent des possibilités inédites dans le domaine de l'analyse du social. Descendu dans les gouffres de son moi, Leiris, quant à lui, ajoute à ces analyses la perspective individuelle qui, centrée

${ }^{38}$ L. Bossi, « L’homme nouveau. Dégénérescence et régénération », [dans :] Les années $1930 \ldots$, p. 55.

39 J. Clair, op. cit., p. 22.

40 A. Dubilet, op. cit., p. 164.

${ }^{41}$ Cette parenté est suggérée par Jean-Claude Monod dans "What's New in Our Current 'International Secularism'? », Leiden Journal of International Law 25, 2012, p. 70 ; et par Frédéric Keck dans « Le sacrifice des insectes. Caillois entre Lévi-Strauss et Bataille », Littérature 170, 2013, p. 32 . 
sur les idiosyncrasies linguistiques et les interstices de la mémoire, incite à dépasser les dichotomies faciles de l'immanence et de la transcendance, du sacré et du profane, pour se tourner vers l'horizon anthropologique. Profondément ancré dans le moment historique mais surtout dans les modèles de perception et l'imaginaire collectif, cet horizon échappe aux schémas binaires des « formes pétrifiées » de la religion et propose ainsi des reformulations du sacré et du politique qui ne peuvent qu'enrichir le discours post-séculier d'aujourd'hui en l'ouvrant à l'une de ses généalogies insoupçonnées.

\title{
TOWARDS THE ANTHROPOLOGICAL FOUNDATIONS \\ OF POST-SECULARISM: THE EXAMPLE OF COLLĖGE DE SOCIOLOGIE
}

\begin{abstract}
Summary
Founded by Georges Bataille, Roger Caillois and Michel Leiris, and conceived as a reaction of leftist Paris intellectuals against fascism, the College of Sociology is an interesting example of the mixture of different types of post-secular thought - a-theological (Bataille), community (Caillois), intimate (Leiris) - imagined as a remedy against the mythical forces handled with brio by the European rights of the 1930s. Already analyzed on the political and discursive level, the College also lends itself to an anthropological analysis in which the post-secular aspect finds a basis in the conception of the "new man," and proves useful in the interpretation of contemporary post-secularism.
\end{abstract}

Key words: Collège de Sociologie, Georges Bataille, Roger Caillois, Michel Leiris, post-secularism, sacred, anthropology. 